\title{
Capacidades organizacionales en las empresas de manufacturas de Cuenca - Ecuador
}

\section{Organizational capacities in the manufacturing companies of Cuenca - Ecuador}

Silvia Raquel Mejía Matute

Universidad del Azuay, Ecuador

smejia@uazuay.edu.ec

(D) https://orcid.org/0000-0003-1256-8165

Luis Gabriel Pinos Luzuriaga

Universidad del Azuay, Ecuador

lpinos@uazuay.edu.ec

iD https://orcid.org/0000-0002-3894-8652

Wazhington Bladimir Proaño Rivera

Universidad del Azuay, Ecuador

wproano@uazuay.edu.ec

https://orcid.org/0000-0003-4959-7410

Erika Belén García Galarza

Universidad del Azuay, Ecuador

ebgarcia@uazuay.edu.ec

D https://orcid.org/0000-0003-4590-3273

Recepción: 04/01/2021 | Aceptación: 10/03/2021 | Publicación: 10/05/2021

\section{Cómo citar (APA, séptima edición):}

Mejía Matute, S. R., Pinos Luzuriaga, L. G., Proaño Rivera, W. B. y García Galarza, E. B. (2021). Capacidades organizacionales en las empresas de manufacturas de Cuenca - Ecuador. Innova Research Journal, 6(2), 133-156. https://doi.org/10.33890/innova.v6.n2.2021.1667

\section{Resumen}

La industria de manufacturas en el Ecuador debe enfrentar el reto de ser más competitiva incrementando su productividad, lo cual requiere una organización empresarial capaz de gestionar eficazmente los recursos, los procesos, y obtener resultados que le permitan aprender y evolucionar. Para ello, se requiere conocer la organización empresarial interna que permita proponer estrategias. Entonces, la pregunta es: ¿cuáles son las principales características de las 
capacidades organizacionales de las empresas manufactureras de Cuenca? Para responder a esta pregunta se aplicó una encuesta utilizando el método probabilístico para determinar la muestra. Se analizaron los datos utilizando estadísticas descriptivas como el método de diferencias y el método de componentes principales para establecer categorías de las capacidades organizativas. Se obtuvo que las empresas manufactureras de Cuenca en promedio le dan un valor de $86.5 \%$ a sus capacidades organizativas y son las grandes las que presentan mayores fortalezas. Los recursos humanos y tecnológicos son importantes en las empresas, pero no suficientes para construir capacidades en las organizaciones, se requiere de coordinación, gestión y control para mantener operativa una tecnología, ampliar los mercados, responder a estrategias para mejorar la producción y disminuir los costos.

Palabras claves: capacidades empresariales; capacidades organizacionales; componentes principales; manufacturas.

\begin{abstract}
The manufacturing industry in Ecuador must face the challenge of being more competitive by increasing its productivity, which requires a business organization capable of effectively managing resources, processes, and obtaining results that will allow it to learn and evolve. For this, it is required to know the internal business organization that allows proposing strategies. So the question is: What are the main characteristics of the organizational capacities of manufacturing companies in Cuenca? To answer this question, a survey was applied using the probabilistic method to determine the sample. Data were analyzed using descriptive statistics such as the difference method and the principal components method to establish categories of organizational capabilities. It was obtained that the manufacturing companies of Cuenca on average give a value of $86.5 \%$ to their organizational capacities and the large ones are the ones that present the greatest strengths. Human and technological resources are important in companies but not enough to develop capacities in organizations, coordination, management and control are required to keep a technology operational, expand markets, and respond to strategies that adapt, grow and innovate before a challenging economic environment to overcome the crisis.
\end{abstract}

Keywords: business capabilities; organizational capacities; main components; manufacturing.

\title{
Introducción
}

El análisis de las capacidades empresariales tiene sus raíces en la economía evolutiva (Nelson y Winter, 1982) que critica las explicaciones de la microeconomía clásica y propone el desarrollo de las capacidades organizativas sobre la economía de la empresa (Knight y Cavusgil, 2004). El propósito es entender su organización interna para reducir la incertidumbre y lo hace a través de una semejanza con el ciclo de la vida. Esta analogía es debida a que se tiene un patrón general y conjunto de posibles caminos que caracterizan su evolución. De la misma manera, se podría decir que las capacidades de la empresa son dinámicas (Schreyogg y Kliesch, 2007) porque el elemento que permite esta constante evolución es el conocimiento y por ello se podrían transformar y crear nuevas capacidades de orden superior que facilitan el aprendizaje sobre nuevos dominios para satisfacer las necesidades del mercado (Ellonen et al., 2011). 
En un estudio realizado para conocer la capacidad empresarial de la industria aeronáutica en México, Hernández (2017) define que: "las capacidades organizacionales son las actividades y habilidades que tienen las empresas para organizar, coordinar, controlar y lograr las tareas, en términos productivos y administrativos, que demanda la empresa de manera eficaz" (p. 69).

Grant (1996) es uno de los primeros autores que analiza a las capacidades organizativas poniendo énfasis en la importancia de los recursos y la integración del conocimiento especializado como fuente para sostener las ventajas competitivas en las empresas. Plantea que, si el conocimiento es un insumo crítico en todos los procesos de producción, si la eficiencia requiere que sea creado y almacenado por individuos en forma especializada, y si la producción requiere la aplicación de muchos tipos de conocimiento especializado, entonces la función principal de la empresa es la integración de conocimiento. La mayoría de las capacidades organizativas requieren la integración de las bases de conocimientos especializados de varias personas y esto se desarrolla en la empresa a través de la rutinización de actividades y la dirección empresarial.

Al hablar de capacidades organizacionales, se habla también de capacidades de gestión o capacidades operativas, ya que estas se refieren a procesos organizacionales, como recursos humanos (calidad, habilidades, competencia); recursos físicos y materiales (máquinas, terrenos y edificios); recursos financieros (dinero y crédito); recursos de información (conocimiento y bases de datos); recursos intelectuales e innovación (patentes, derechos de autor y diseño) (Boonpattarakan, 2012).

Ciertamente, la administración, el control, la coordinación y la capacidad de llevar a cabo un conjunto de actividades en la organización está directamente correlacionado con el grado de desarrollo de las capacidades (Degravel, 2011). De esta manera, se considera necesario conocer las capacidades organizacionales para comprender el funcionamiento de las empresas y proponer estrategias que les permitan ser más competitivas.

Cabe recalcar que las empresas buscan generar y sobre todo desarrollar sus capacidades con el fin de adaptarse adecuada y rápidamente a los volátiles requerimientos del mercado así, al acumular y renovar capacidades, se permite administrar y encaminar el conocimiento, la innovación y el aprendizaje, con lo cual se intensifica la competitividad entre las distintas empresas en el mercado (García et al., 2014).

La ciudad de Cuenca se ubica en el Austro del Ecuador. Antes de 1950 su industria manufacturera se caracterizaba por ser eminentemente artesanal, pero su desarrollo industrial comenzó a partir de la estrategia de sustitución de importaciones promovida por la CEPAL en los años 50 del siglo pasado y aplicada en los años 70 en el país. En aquel entonces, se establecieron condiciones para el desarrollo de la industria como la construcción de parques industriales y una política de fomento industrial que duró poco.

Ecuador es un país dolarizado desde el año 2000, esta situación le trae ventajas de estabilidad monetaria y desventajas cuando de competitividad se trata, pues cuando se aprecia el dólar se encarece su producción y por ello requiere tener alta productividad. La industria manufacturera está muy afectada por la competencia de productos importados más baratos; por ello, es importante conocer cuáles son sus capacidades empresariales para enfrentar al mercado 
global. Entonces, la pregunta es: ¿cuáles son las principales características de las capacidades organizacionales de las empresas manufactureras de Cuenca en 2019?

El objetivo de este estudio es conocer las características de las capacidades organizacionales de las empresas de manufacturas de Cuenca en 2019 con la finalidad de aportar al análisis de las estrategias que encausan su fomento y dinámica hacia la innovación. Para cumplir con este objetivo en primer lugar, se presenta una revisión de la literatura sobre el tema, luego se muestra la metodología que está basada en la aplicación de una encuesta con el método de muestreo probabilístico y la estrategia de análisis es basada en el estudio de las diferencias y los componentes principales. Luego, se presentan los resultados donde se establece que, las empresas de Cuenca tienen importantes capacidades organizacionales. Por último, se discuten los resultados y arriba a las conclusiones.

\section{Marco teórico}

\section{Las Capacidades Organizacionales}

Grant (1996) está entre los primeros autores que aborda a las capacidades organizativas en su clásico artículo titulado "Prospering in Dynamically-Competitive Environments: Organizational Capability as Knowledge". Su análisis pone énfasis en la importancia del conocimiento y su integración en las actividades y estrategias de la empresa como una fuente de capacidades que permitan enfrentar el cambiante entorno empresarial. Entonces se considera que, las capacidades organizacionales están correlacionadas directamente con el conocimiento, el cual puede ser explícito y tácito.

El primero involucra muy pocos problemas de integración porque está inherente en la comunicación. La integración del conocimiento explícito se ha facilitado por medio de las tecnologías de información, debido a que puede ser codificado, comunicado, asimilado, almacenado y recuperado a través de manuales, procedimientos mecanizados, actividades planeadas como permanentes para producir productos o servicios estandarizados y reconocidos por el saber hacer de la empresa. En cambio, el conocimiento tácito, no puede expresarse explícitamente ni codificarse en forma escrita (Nonaka y Takeuchi, 2000). Es considerado como un problema preocupante al momento de integrarlo porque está referido a la experiencia del equipo, y su grado de especialización (Grant, 1996).

Por ello, se puede decir que la esencia de la capacidad organizacional está en la integración de los conocimientos especializados (Teece et al., 1997). Se requiere liderazgo y comunicación fluida para transferir el conocimiento e integrarlo a las actividades y estrategias de la empresa. Entonces, de acuerdo al nivel de integración del conocimiento se pueden reconocer en las organizaciones "las capacidades operativas" (Acevedo y Albornoz, 2019), relacionadas con las tareas automatizadas, estandarizadas, rutinarias. Por otra parte, en una jerarquía superior se encuentran la gama de conocimientos especializados en la fabricación misma de los productos u otras capacidades específicas como las empresariales donde se amalgama el mercadeo, las finanzas y la gestión en general. A continuación, y subiendo en la escala se tiene en el estatus más avanzado 
a las capacidades multifuncionales que requieren de un conocimiento especializado que inventa, diseña y crea valor agregado como el desarrollo de nuevos productos y procesos.

Para Martínez y Espinosa (2017), las capacidades organizacionales implican un proceso de construcción y apropiación dado que el aprendizaje es un proceso histórico que está influenciado por las grandes empresas, es decir el impacto que tienen las trayectorias tecnológicas pasadas en el desarrollo de las nuevas capacidades y donde el empresario rompe con el modelo lineal de la innovación y logra integrar en el proceso de innovación.

Como se ha denotado, el conocimiento conjunto de una organización se refiere a la capacidad de la empresa para aprender y utilizar las relaciones entre factores y medios informativos para lograr los fines previstos en la organización (Autio et al., 2000). Así, la construcción de capacidades no es sólo un proceso de acumulación sino también de transformación porque podrían considerarse como capacidades de orden superior que facilitan el aprendizaje sobre nuevos dominios, crean nuevas combinaciones de activos y crean nuevas capacidades para satisfacer las necesidades del mercado (Ellonen et al., 2011). Por ello, son factores claves la adquisición de conocimientos, el intercambio, la formación de redes y los procesos de innovación (Jurksiene et al., 2016).

Las capacidades organizacionales tienen dos aspectos principales, por un lado, el carácter cambiante del entorno empresarial; y, por otro lado, la gestión estratégica para adaptar, integrar y reconfigurar adecuadamente las capacidades basadas en el conocimiento hacia el entorno cambiante. Para conseguirlas es importante resaltar sus fuentes, así se tiene el caso de la construcción de mejores y renovados productos y servicios, el suministro de productos con precios competitivos, y centrarse en el desarrollo de las capacidades tecnológicas, para mejorar la competitividad (Nonaka y Takeuchi, 2000; Boonpattarakan, 2012).

Como se evidencia, la administración, el control, la coordinación y la capacidad de llevar a cabo un conjunto de actividades en la organización está directamente relacionada con el grado de desarrollo de las capacidades (Degravel, 2011; Hernández, 2017).

Así pues, las empresas buscan generar y sobre todo desarrollar sus capacidades, con el propósito de adaptarse adecuada y rápidamente a los volátiles requerimientos del mercado, así al acumular y renovar capacidades, se permite administrar y encaminar el conocimiento, la innovación y el aprendizaje, con lo cual se intensifica la competitividad entre las distintas empresas en el mercado (García et al., 2014).

Las capacidades organizacionales, son parte de la estructura de las entidades sociales que representan a una colectividad y están destinadas a resolver problemas, de esta forma evolucionan a través del tiempo debido a que desarrollan procesos de aprendizaje (consecuentemente se genera un componente dinámico intrínseco); a la vez, representan una forma distintiva de combinar y asignar recursos en la organización (Helfat y Peteraf, 2003; Carnahan et al., 2010).

Por lo acotado, es necesario concebir a las capacidades organizacionales como una actividad colectiva, por medio de la cual, la empresa realiza sus rutinas con el mejor método de funcionamiento de sus procesos y actividades (con integración del conocimiento), para alcanzar la 
ventaja competitiva, y posteriormente convertir a estas actividades en procesos sistémicos y mecánicos dentro de la empresa (Killen et al., 2008).

Sin embargo, la visión basada en recursos ha sido muy criticada porque gira en torno al hecho de dar poca relevancia a la dinámica del entorno, generando por una parte en sus postulados un carácter muy estático en la relación organización-entorno. Entonces, la perspectiva de la capacidad dinámica amplía el argumento de la visión basada en recursos al abordar cómo se pueden crear recursos valiosos, raros, difíciles de imitar e imperfectamente sustituibles y cómo se puede actualizar el stock actual de recursos valiosos en entornos cambiantes (Ambrosini y Bowman, 2009).

Para implantar las capacidades organizacionales en la empresa hay que entenderlas también como capacidades dinámicas que evolucionan por medio del conocimiento explícito y la acumulación de experiencia (Zollo y Winter, 2002).

La literatura sobre capacidades de gestión dinámica se ha desarrollado durante la década de los noventa a partir de los estudios de Teece et al. (1997) quien las define como: "la capacidad de la empresa para integrar, construir y reconfigurar las competencias internas y externas para enfrentar un entorno cambiante. Capacidades que reflejan las habilidades de la organización para alcanzar nuevas e innovadores formas de ventajas competitivas" (p. 517). Sin duda, el campo ha avanzado considerablemente desde entonces y se ha centrado en tres pilares. El primero, se refiere a la cognición gerencial; el segundo es el capital social gerencial y el tercero el capital humano gerencial (Helfat y Martin, 2015).

Zapata y Mirabal (2018), proponen un modelo de capacidades dinámicas donde se integran una serie de conceptos y componentes de modelos de capacidades dinámicas ofrecidos en la literatura: capacidad de adaptación, capacidad de innovación, capacidad organizacional, capacidad relacional, capacidad de absorción, capacidad de detección y capacidad de integración.

En este trabajo se ha puesto énfasis en la capacidad de las empresas para integrar el conocimiento con el propósito de responder a un mercado cambiante y que en la actualidad enfrenta un entorno macroeconómico de crisis. 


\section{Figura 1}

Integración del conocimiento, desde el complejo hacia el simple

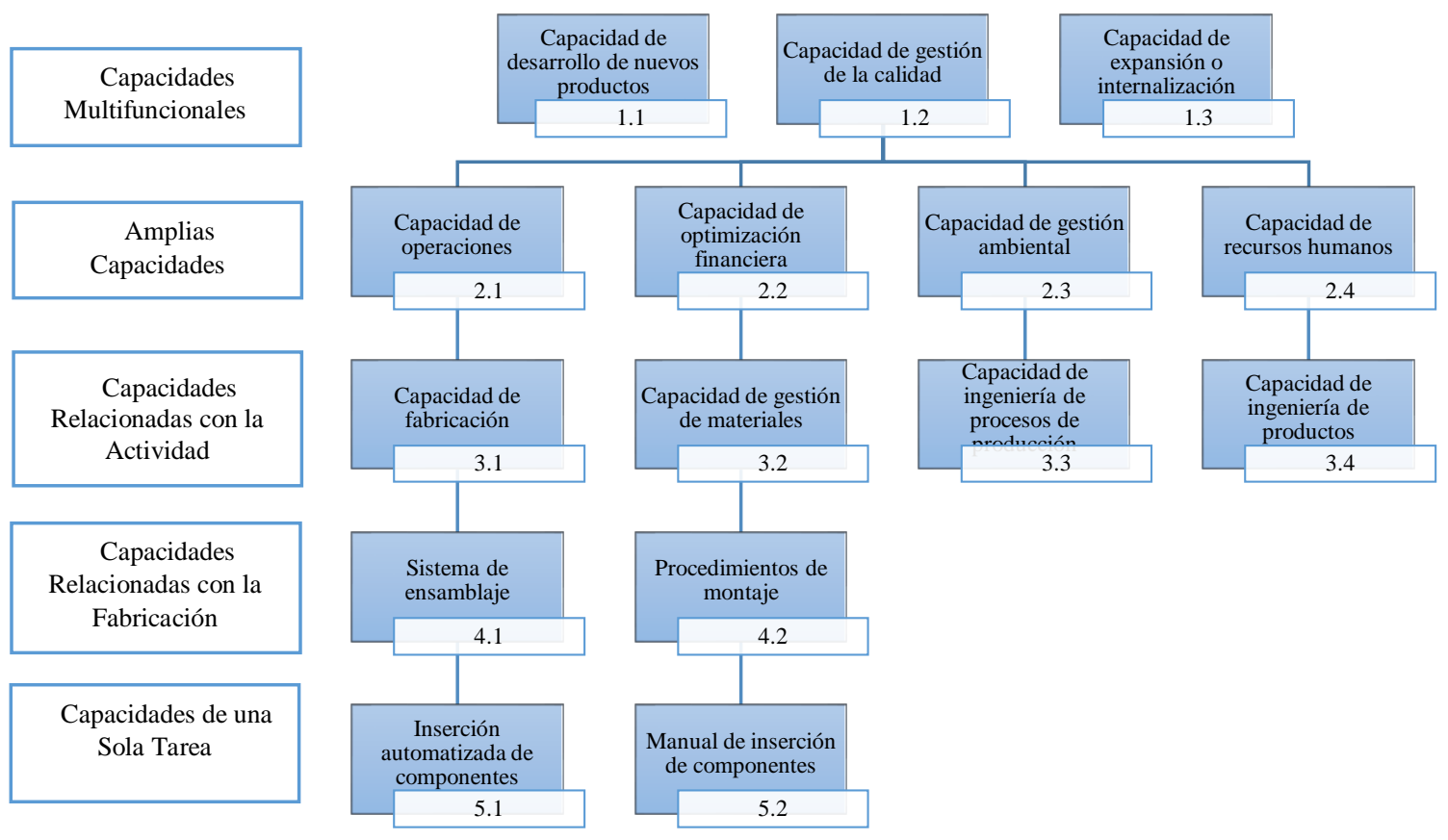

Nota: Elaboración propia.

En la figura 1, se aprecia el proceso de integración del conocimiento, desde las capacidades multifuncionales que conllevan conocimiento especializado, hasta las capacidades simples con conocimientos básicos generales situados en el seguimiento de procedimientos previamente establecidos.

\section{Metodología}

Esta investigación se llevó a cabo en la ciudad de Cuenca - Ecuador con el objetivo de conocer las capacidades organizacionales de las empresas pequeñas, medianas y grandes que pertenecen al sector $\mathrm{C}$ de la industria manufacturera según la Clasificación Industrial Internacional Uniforme-CIIU. Para ello, se consideró pertinente utilizar una metodología cuantitativa mediante la aplicación de una encuesta.

Se elaboró un instrumento de recolección de datos con 37 preguntas divididas en 8 secciones. La primera sección, se refiere a las características del establecimiento y tiene preguntas relacionadas con la ubicación, el tamaño y la actividad económica. Luego, se tiene una sección referida a las características de quienes administran o son propietarios con el propósito de conocer y cuantificar aspectos como el nivel de educación, experiencia, habilidades y la frecuencia de la capacitación durante un año. De la misma manera, la siguiente sección indaga sobre las capacidades del talento humano tanto en el área productiva como administrativa. Finalmente, se 
tiene un bloque de preguntas sobre los resultados de las capacidades organizacionales, sobre todo de aquellas que permiten integrar el conocimiento en la empresa a través de rutinas, dirección, estrategia empresarial y transmisión y creación de ideas. En la tabla 1 se exponen las variables, indicadores y tipo de respuestas esperadas ya sea con dos categorías como sí o no, y también con una escala del 1 al 5 o según la categorización de la variable.

En esta investigación se consideró como unidad de observación a las empresas seleccionadas que son la fuente de información ya que se pretende conocer sus características y capacidades. De esta manera se tiene que la unidad de respuesta corresponde a la persona informante que proporcionó los datos.

\section{Tabla 1}

Categorías y variables utilizadas

\begin{tabular}{|c|c|c|}
\hline Categoría & Variable o indicador & Valor \\
\hline \multirow{6}{*}{$\begin{array}{l}\text { Capacidades del } \\
\text { recurso humano } \\
\text { directivo, } \\
\text { administrativo y } \\
\text { producción. }\end{array}$} & $\begin{array}{l}\text { Escolaridad: } \\
\text { 1. Ninguno } \\
\text { 2. Centro de alfabetización } \\
\text { 3. Primaria } \\
\text { 4. Secundaria } \\
\text { 5. Tecnológico } \\
\text { 6. Superior } \\
\text { 7. Postgrado }\end{array}$ & $\begin{array}{l}\text { Los valores dependen de la } \\
\text { respuesta y van del } 1 \text { al } 7 .\end{array}$ \\
\hline & $\begin{array}{l}\text { Años de experiencia: (¿si tiene un año y medio de } \\
\text { experiencia, en qué categoría entra? } \\
\text { 1. Entre cero a un año/Hasta un año } \\
\text { 2. Entre dos a cinco años (se suele decir: de } 2 \text { a } 5 \text {, o } \\
\text { entre } 2 \text { y } 5 \text {. No la combinación de ambas: entre } 2 \text { a 5) } \\
\text { 3. Más de cinco años }\end{array}$ & $\begin{array}{l}\text { Los valores dependen de la } \\
\text { respuesta y van del } 1 \text { al } 3 \text {. }\end{array}$ \\
\hline & $\begin{array}{l}\text { Pertenencia a una organización: } \\
\text { 1. Cámara de Comercio de Cuenca } \\
\text { 2. Cámara de Comercio Ecuatoriana Americana } \\
\text { 3. Cámara de la Industria, Producción y Empleo } \\
\text { 4. Cámara de la Pequeña Industria } \\
\text { 5. Otra. Escriba ¿Cuál? } \\
\text { 6. No es miembro de ningún gremio u organización } \\
\text { empresarial } \\
\text { 7. Pertenece a más de una organización }\end{array}$ & $\begin{array}{l}\text { Los valores dependen de la } \\
\text { respuesta y van del } 1 \text { al } 7 .\end{array}$ \\
\hline & $\begin{array}{l}\text { Innovación organizacional: } \\
\text { 1. Nuevas prácticas en la organización de } \\
\text { procedimientos } \\
\text { 2. Nuevos métodos para la organización de } \\
\text { responsabilidades laborales y toma de decisiones } \\
\text { 3. Nuevos métodos en la organización de relaciones } \\
\text { externas }\end{array}$ & 1. $\mathrm{Si}, 2 . \mathrm{No}$ \\
\hline & Nuevas formas de contratación de personal & 1. Si, 2. No \\
\hline & $\begin{array}{l}\text { Inversión en capacitación a obreros o contratación } \\
\text { de asesoría técnica }\end{array}$ & 1. $\mathrm{Si}, 2 . \mathrm{No}$ \\
\hline
\end{tabular}




\begin{tabular}{|c|c|c|}
\hline Categoría & Variable o indicador & Valor \\
\hline \multirow{10}{*}{$\begin{array}{l}\text { Capacidades } \\
\text { organizacionales } \\
\text { específicas }\end{array}$} & Aumento de la gama de bienes o servicios & \multirow{10}{*}{$\begin{array}{l}\text { La importancia dada a cada una } \\
\text { de las variables, en la escala de } \\
\text { valores donde: } \\
5 \text { significa muy importante, } \\
4 \text { se refiere a importante, } \\
3 \text { indica moderada importancia, } \\
2 \text { es poca importancia, y } \\
1 \text { implica ninguna importancia. }\end{array}$} \\
\hline & Mejora de calidad de bienes o servicios & \\
\hline & $\begin{array}{l}\text { Entrada a nuevos mercados o aumento de la } \\
\text { participación de mercado }\end{array}$ & \\
\hline & $\begin{array}{l}\text { Mejoras en la flexibilidad para producir bienes o } \\
\text { servicios (mejoras en los tiempos) }\end{array}$ & \\
\hline & $\begin{array}{l}\text { Reducción de costos laborales por unidad de } \\
\text { producción }\end{array}$ & \\
\hline & Reducción del impacto ambiental & \\
\hline & Mejoras en la seguridad y salud del trabajador & \\
\hline & $\begin{array}{l}\text { Reducción de costos de materias primas y energía } \\
\text { por unidad de producción }\end{array}$ & \\
\hline & $\begin{array}{l}\text { Mejoras en la capacidad instalada para producir } \\
\text { bienes o servicios (volumen máximo de producción a } \\
\text { alcanzar en un tiempo determinado considerando la } \\
\text { disponibilidad de recursos) }\end{array}$ & \\
\hline & Reemplazo de productos o procesos obsoletos & \\
\hline
\end{tabular}

Elaboración propia a partir de la información extraída de las encuestas Estado de las Empresas en Cuenca, 2020

Se utilizó como marco muestral al directorio proporcionado por la Superintendencia de Compañías y Seguros (2019), correspondiente al sector societario en la ciudad de Cuenca, en situación legal activa del sector industrial manufacturero, que presentan balances dividido en grupos de acuerdo a la clasificación industrial internacional uniforme (CIIU 4.0) nivel 2. Las empresas que cumplen estas características fueron 328 en el año 2019, y son las que pasan a representar nuestro universo de estudio.

La cobertura geográfica incluye a todas aquellas empresas ubicadas en la ciudad de Cuenca, provincia del Azuay en Ecuador, y, la cobertura temática fue a nivel de las actividades económicas según la CIIU 4.0 nivel 2 y la clasificación por tamaño de las empresas considerando el número de trabajadores y el monto anual de ingresos, predominando siempre los ingresos sobre el número de trabajadores. A continuación, se presenta la clasificación de las empresas industriales de la ciudad de Cuenca según el tamaño:

\section{Tabla 2}

Clasificación según tamaño de la empresa

\begin{tabular}{llc}
\hline & Tamaño empresa & Número de compañías \\
\hline 1. & Microempresa & 148 \\
2. & Pequeña & 119 \\
3. & Mediana & 43 \\
4. & Grande & 18 \\
\hline Total & & $\mathbf{3 2 8}$ \\
\hline
\end{tabular}


Información extraída a partir de datos presentados por la Superintendencia de Compañías, Valores y Seguros, 2019.

Se utilizó el "muestreo aleatorio estratificado" (Lind et al, 2012, p. 270). La población es 328 empresas registradas como activas por tamaño y actividad en la industria manufacturera con un nivel de confianza del $95 \%$ y un margen de error del $5 \%$. El resultado fue 178 empresas. Empero, se tuvo que recalcular el tamaño de la muestra debido a los problemas en la recolección de datos por causa de cambios de domicilio, otros números telefónicos de contacto para realizar la entrevista, empresas en proceso de liquidación o que ya habían respondido otras encuestas y mostraron negativas para responder. Para establecer un nuevo tamaño de la muestra se amplió el margen de error a $6 \%$ y se disminuyó el nivel de confianza al $90 \%$, se obtuvo como resultado que el nuevo tamaño de la muestra fue de 122 encuestas. El método para distribuir la muestra está en relación al número de empresas que existe en el universo de estudio considerando las características de actividad económica CIIU y tamaño de la empresa. El levantamiento de información se realizó el último trimestre de 2019, para lo cual la tabla 3 muestra en detalle.

\section{Tabla 3}

Distribución de la muestra por tamaño y según actividad

\begin{tabular}{|c|c|c|c|c|c|}
\hline \multirow{2}{*}{$\begin{array}{c}\text { ACTIVIDAD } \\
\text { ECONÓMICA } \\
\text { SEGÚN CIIU N² }\end{array}$} & \multicolumn{4}{|c|}{ TAMAÑO EMPRESA } & \multirow[b]{2}{*}{$\begin{array}{l}\text { TOTAL NÚMERO } \\
\text { DE EMPRESAS }\end{array}$} \\
\hline & $\begin{array}{l}\text { GRAND } \\
\text { E }\end{array}$ & $\begin{array}{c}\text { MEDIAN } \\
\text { A }\end{array}$ & $\begin{array}{l}\text { MICROEMPRES } \\
\text { A }\end{array}$ & PEQUEÑA & \\
\hline $\mathrm{C} 10$ & 1 & 2 & 9 & 4 & 16 \\
\hline $\mathrm{C} 11$ & 0 & 1 & 3 & 1 & 5 \\
\hline $\mathrm{C} 13$ & 0 & 0 & 2 & 1 & 3 \\
\hline C14 & 0 & 2 & 3 & 5 & 10 \\
\hline $\mathrm{C} 15$ & 0 & 0 & 1 & 1 & 3 \\
\hline C16 & 0 & 0 & 1 & 1 & 3 \\
\hline $\mathrm{C} 17$ & 0 & 0 & 1 & 0 & 2 \\
\hline $\mathrm{C} 18$ & 0 & 1 & 4 & 3 & 7 \\
\hline C19 & 0 & 0 & 0 & 0 & 1 \\
\hline $\mathrm{C} 20$ & 0 & 1 & 6 & 3 & 10 \\
\hline $\mathrm{C} 21$ & 0 & 0 & 4 & 2 & 6 \\
\hline $\mathrm{C} 22$ & 1 & 1 & 1 & 3 & 5 \\
\hline $\mathrm{C} 23$ & 2 & 2 & 5 & 4 & 12 \\
\hline $\mathrm{C} 24$ & 0 & 0 & 1 & 2 & 4 \\
\hline $\mathrm{C} 25$ & 0 & 1 & 3 & 4 & 8 \\
\hline $\mathrm{C} 26$ & 0 & 0 & 3 & 1 & 4 \\
\hline $\mathrm{C} 27$ & 0 & 0 & 0 & 1 & 2 \\
\hline $\mathrm{C} 28$ & 0 & 0 & 1 & 0 & 3 \\
\hline $\mathrm{C} 29$ & 0 & 0 & 1 & 1 & 2 \\
\hline
\end{tabular}




\begin{tabular}{|c|c|c|c|c|c|}
\hline \multirow{2}{*}{$\begin{array}{c}\text { ACTIVIDAD } \\
\text { ECONÓMICA } \\
\text { SEGÚN CIIU N }{ }^{\circ} 2\end{array}$} & \multicolumn{4}{|c|}{ TAMAÑO EMPRESA } & \multirow[b]{2}{*}{$\begin{array}{l}\text { TOTAL NÚMERO } \\
\text { DE EMPRESAS }\end{array}$} \\
\hline & $\begin{array}{l}\text { GRAND } \\
\text { E }\end{array}$ & $\begin{array}{l}\text { MEDIAN } \\
\text { A }\end{array}$ & $\begin{array}{c}\text { MICROEMPRES } \\
\text { A }\end{array}$ & PEQUEÑA & \\
\hline $\mathrm{C} 30$ & 0 & 0 & 0 & 1 & 2 \\
\hline $\mathrm{C} 31$ & 0 & 2 & 2 & 3 & 7 \\
\hline $\mathrm{C} 32$ & 0 & 1 & 2 & 2 & 5 \\
\hline $\mathrm{C} 33$ & 0 & 0 & 1 & 3 & 4 \\
\hline Total & 7 & 16 & 55 & 44 & 122 \\
\hline
\end{tabular}

Elaboración propia a partir de la información extraída de las encuestas Estado de las Empresas en Cuenca, 2020

La estrategia de análisis implementada fue, en primer lugar, una descripción de los resultados de las variables e indicadores. En segundo lugar, se realizó un análisis de componentes principales para establecer las categorías de las capacidades organizacionales, y, finalmente, se presentan a estas categorías obtenidas por tamaño de empresa.

El análisis descriptivo permite dar cuenta de las características de los recursos humanos tanto de los directivos como del personal que trabaja en las áreas de administración y producción. Además, utilizando promedios simples se presenta una metodología basada en las diferencias para comparar, por un lado, el promedio del estado actual de las capacidades organizativas de las empresas con la mejor situación que se obtuvo de la empresa " $\mathrm{X}$ ", $\mathrm{y}$, por otro lado, se tiene la peor situación que corresponde a la empresa "Y". Así, este análisis permite mostrar fortalezas y debilidades en torno a las capacidades organizacionales de las empresas de Cuenca en 2019.

Luego con el bloque de preguntas relacionadas específicamente con las capacidades organizacionales se realizó una evaluación de resultados para probar si son estadísticamente representativos a través de una medida de consistencia interna basada en la correlación inter elementos promedio como es el Alfa de Cronbach. Esta es una medida cuyo promedio ideal es que se encuentre cerca de 1 .

Las preguntas consideradas para el análisis de Cronbach poseen casos eliminados y validos por lo que están un gran número de espacios en blanco. Considerando el análisis correlacional inter elementos, el ítem que menos aporta es el primero con una correlación de 0.487 al eliminar este primer elemento el alfa subió de 0.845 a 0.856 .

Luego de esta evaluación interna se realizó un análisis de componentes principales para evidenciar estadísticamente la relación entre las variables que permitan establecer las categorías de capacidades organizacionales y conocer la ponderación que cada variable aporta a través de los resultados de varianza y auto valor que arroja este análisis.

De las diez variables de la encuesta se tiene 113 respuestas de 122, la media de las respuestas de una escala entre 1 a 5 (siendo 5 muy importante y 1 significa ninguna importancia), y, la desviación estándar. 
Se considera pertinente analizar la correlación entre estas variables y con ello establecer componentes principales, es decir categorías que se forman entre variables con alta correlación y que indican están midiendo un mismo factor bajo diferentes puntos de vista, en otras palabras se reduce el número de variables y se transforman en un nuevo grupo de variables que están correlacionadas en otro conjunto de variables no correlacionadas entre sí, a estas las llamaremos los componentes principales o categorías estadísticamente representativas.

Se realizó una matriz de correlaciones de variables y se obtuvo un determinante de 0.191 que es diferente de 1, que significa que para probar las correlaciones entre las variables deben ser diferentes de cero de modo estadísticamente significativo, con el resultado de 0.191 se comprueba que la determinante de la matriz es distinta a uno, es otras palabras la matriz de correlaciones es distinta a la de identidad. Si las variables están correlacionadas, habrá muchos valores altos (en valor absoluto) fuera de la diagonal principal de correlaciones, por tanto, el determinante será menor a 1. (Si las variables están incorrelacionadas, el determinante máximo de la matriz será de 1). Para comprobar se utilizó el test de Bartlett con la siguiente hipótesis:

Ho: El determinante de la matriz de correlaciones es igual a 1

H1: El determinante de la matriz de correlaciones es diferente a 1

El determinante de una matriz nos da una idea de la correlación generalizada de todas las variables, se basa en la distribución Chi Cuadrado, por tanto, la prueba de esfericidad de Bartlett contrasta si la matriz de correlaciones es una matriz identidad, lo que indicaría que el modelo factorial es inadecuado. En la tabla 4, se evidencia que la prueba de Bartlett nos muestra un valor Chi Cuadrado de 178.385, con un p valor de cero, razón por la cual se rechaza la hipótesis nula Ho-.

\section{Tabla 4}

Prueba de KMO y Bartlett

\begin{tabular}{lll}
\hline \multicolumn{2}{c}{ Medida Kaiser-Meyer-Olkin de adecuación de muestreo } & 0.534 \\
\hline \multirow{2}{*}{ Prueba de esfericidad de } & Aprox. Chi-cuadrado & 178.385 \\
Bartlett & gl & 45 \\
& Sig. & 0.000 \\
\hline
\end{tabular}

Para aplicar el método de extracción de los componentes principales se usó el criterio de Kaiser, que consiste en conservar los factores cuyo autovalor asociado sea mayor a 1. Con este criterio se obtuvo 5 componentes, con una varianza explicada de $73.415 \%$.

En la tabla 5, se observan las 10 variables o componentes analizados y el resultado extraído de los componentes principales, donde se establecen 5 categorías. 


\section{Tabla 5}

Análisis de Componentes Principales

\begin{tabular}{|c|c|c|c|c|c|c|c|c|c|}
\hline \multirow[b]{2}{*}{$\begin{array}{l}\text { Compo } \\
\text { nente }\end{array}$} & \multicolumn{3}{|c|}{ Autovalores iniciales } & \multicolumn{3}{|c|}{$\begin{array}{l}\text { Sumas de extracción de } \\
\text { cargas al cuadrado }\end{array}$} & \multicolumn{3}{|c|}{$\begin{array}{c}\text { Sumas de rotación de cargas } \\
\text { al cuadrado }\end{array}$} \\
\hline & Total & $\begin{array}{c}\% \text { de } \\
\text { varianza }\end{array}$ & $\begin{array}{c}\% \\
\text { acumula } \\
\text { do }\end{array}$ & Total & $\begin{array}{c}\% \text { de } \\
\text { varianz } \\
\text { a }\end{array}$ & $\begin{array}{c}\% \\
\text { acumul } \\
\text { ado }\end{array}$ & Total & $\begin{array}{c}\% \text { de } \\
\text { varianz } \\
\text { a }\end{array}$ & $\begin{array}{c}\% \\
\text { acumula } \\
\text { do }\end{array}$ \\
\hline 1 & 2.270 & 22.703 & 22.703 & 2.270 & 22.703 & $\begin{array}{c}22.70 \\
3\end{array}$ & 1.850 & 18.497 & 18.497 \\
\hline 2 & 1.661 & 16.615 & 39.318 & 1.661 & 16.615 & $\begin{array}{c}39.31 \\
8\end{array}$ & 1.581 & 15.808 & 34.305 \\
\hline 3 & 1.302 & 13.021 & 52.339 & 1.302 & 13.021 & $\begin{array}{c}52.33 \\
9\end{array}$ & 1.400 & 14.005 & 48.310 \\
\hline 4 & 1.100 & 11.005 & 63.344 & 1.100 & 11.005 & $\begin{array}{c}63.34 \\
4\end{array}$ & 1.316 & 13.157 & 61.467 \\
\hline 5 & 1.007 & 10.071 & 73.415 & 1.007 & 10.071 & $\begin{array}{c}73.41 \\
5\end{array}$ & 1.195 & 11.948 & 73.415 \\
\hline 6 & .736 & 7.355 & 80.770 & & & & & & \\
\hline 7 & .617 & 6.170 & 86.940 & & & & & & \\
\hline 8 & .563 & 5.632 & 92.572 & & & & & & \\
\hline 9 & .394 & 3.936 & 96.508 & & & & & & \\
\hline 10 & .349 & 3.492 & $\begin{array}{l}100.00 \\
0\end{array}$ & & & & & & \\
\hline
\end{tabular}

Elaboración Propia a partir de la encuesta Estado de las Empresas en Cuenca, 2020

Las nuevas variables son combinaciones lineales de las primeras y se construyen de acuerdo al orden de importancia en cuanto a la variabilidad total que recogen de la muestra. Entonces, el análisis de componentes principales ha clasificado el conjunto de variables en 5 componentes, los cuales explican el $73.41 \%$ de la varianza. El componente 1, "Capacidad de ampliación y mercadeo"; el segundo se refiere a "La capacidad de cambios tecnológicos de productos o procesos"; el tercero a la "Capacidad de optimización laboral": el cuarto a las "Capacidades relacionadas con la reducción de costos y mejoras de la producción" y finalmente, el quinto tiene que ver con la "Capacidad de mejorar la calidad de los productos".

\section{Resultados}

Como fue dicho en el apartado de la metodología se realizó una encuesta cuya unidad de análisis fueron las empresas activas registradas en la Superintendencia de Compañías como sector de manufacturas.

Los resultados que se presentan a continuación están organizados en tres partes, la primera corresponde a las características generales de las empresas investigadas, la segunda corresponde a 
las características organizacionales que están en relación con el talento humano tanto en el área de administración como de producción y la tercera parte corresponde a las capacidades organizacionales medidas a través de variables que dan cuenta del grado de importancia que los directivos empresariales les dan.

Se encontró que de la muestra de 122 empresas analizadas con $90 \%$ de confianza y $6 \%$ de error, en la ciudad de Cuenca en el año 2019. Como características generales de estas empresas se tiene que, el $76.2 \%$ están inmersas en el proceso productivo y la comercialización, en cambio el $18.9 \%$ solo se ocupa de la producción para entregar a otras empresas que se encargan de la venta de los productos. Los principales clientes de las empresas analizadas son consumidores finales $(68.9 \%)$, los intermediarios $(27 \%)$, supermercados $(1.6 \%)$, tiendas $(0.8 \%)$ y otros $(1.6$ $\%)$. El principal mercado para la comercialización es con cobertura local (68.9\%), el nacional (25.4\%), el mercado regional (4.1\%) y el mercado internacional (1.6\%). La principal motivación para su constitución fue el emprendimiento familiar (El 78.7\%).

Respecto a los resultados relacionados con los recursos humanos, se encontró que $57.4 \%$ de gerentes propietarios son hombres y $40.2 \%$ son mujeres, $2.4 \%$ no contestaron. Se preguntó cuántos años de experiencia cumplidos tenían en ese negocio y se obtuvo que el $70.5 \%$ tiene entre 2 a 5 años, seguido de los más experimentados con más de 5 años (17.5\%) y menor a 1 año (10.6 $\%$ ). Además, se conoció que el $91.8 \%$ de los directivos, gerentes o propietarios realizaron cursos de capacitación, la mayoría realizó entre 2 a 5 cursos específicos en el último año.

En la figura 2, se puede observar el porcentaje de empresas con el nivel de instrucción de los directivos, donde se recalca que la mayoría tiene nivel de instrucción superior.

\section{Figura 2}

Porcentaje de empresas según el nivel de instrucción de sus gerentes o propietarios

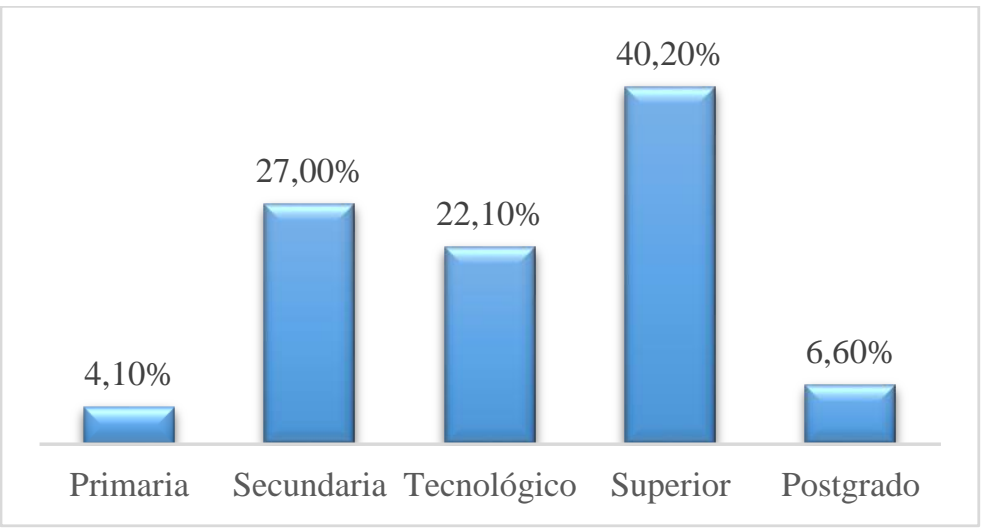

Nota: Elaboración propia a partir de la Encuesta a Empresas Manufactureras de Cuenca, 2020.

Dentro de las características organizacionales es importante que los empresarios estén representados, por ello se les preguntó a cuáles de las siguientes organizaciones pertenecen y respondieron lo siguiente: Cámara de Comercio de Cuenca (27\%), no pertenecen a ningún gremio 
u organización empresarial (26.2\%), Cámara de la Pequeña Industria (20.5\%), otras organizaciones $(3.3 \%)$, pertenece a más de una organización $(2.5 \%)$ y a la Cámara de la Industria, Producción y Empleo $(0.8 \%)$.

Por otro lado, el $76.2 \%$ de los entrevistados consideran que es muy importante el incremento de las ventas para que exista un crecimiento significativo en su negocio. Así mismo, para la sostenibilidad de los establecimientos, el $39.3 \%$ de estas organizaciones, han buscado financiamiento en los bancos privados de la ciudad y el país.

El $48.4 \%$ de las empresas manufactureras cuencanas, tienen alrededor de 10 a 49 empleados en sus negocios. El nivel de instrucción de empleados del área administrativa o de ventas, y del área productiva se encuentra en la figura 3, donde se recalca que la mayoría tienen estudios secundarios.

\section{Figura 3}

Porcentaje de empresas según el nivel de instrucción que predomina en el personal administrativo y de producción

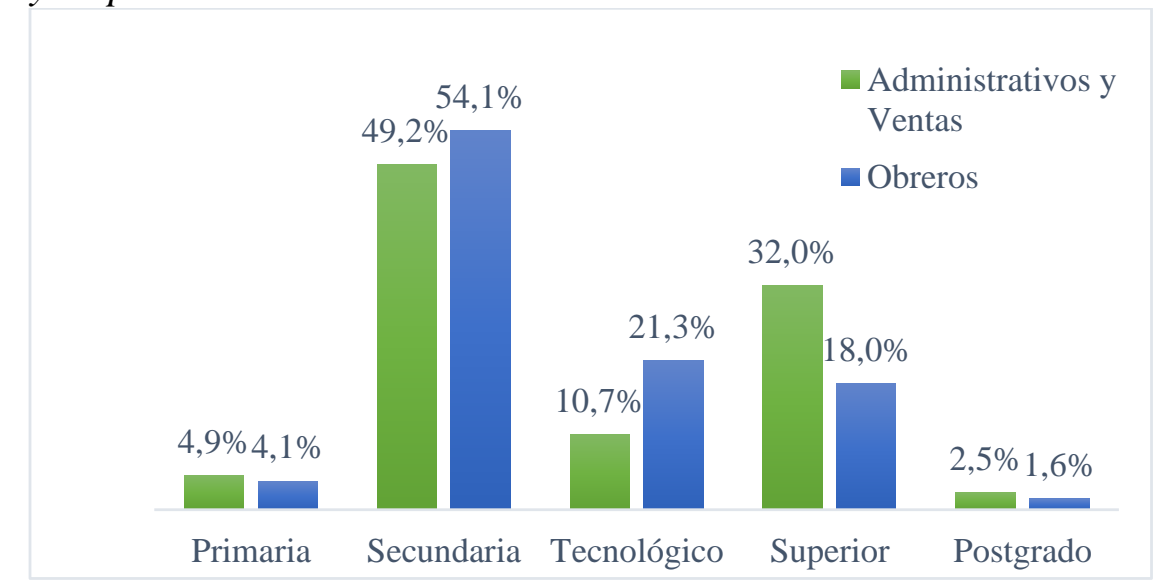

Nota: Elaboración propia a partir de la Encuesta a Empresas Manufactureras de Cuenca, 2020.

El $93.4 \%$ de los directivos de las empresas manufactureras investigadas en la ciudad de Cuenca, consideran que de ser posible utilizarían nuevas formas de contratación del personal, tales como: empleados que ofrecen sus servicios por medio de aplicaciones, empleados a tiempo parcial o empleados semiautónomos, lo que quiere decir que el empresariado de Cuenca se acogería a nuevas formas de demanda laboral y el uso de las nuevas Tecnologías de la Información y Comunicación -TIC’s-. También, las empresas están invirtiendo en el desarrollo de capacitaciones para sus obreros, así ocurrió en $91.8 \%$ de las empresas analizadas.

A más de los recursos humanos para la construcción de capacidades empresariales es importante contar con recursos que permitan integrar el conocimiento y aprendizaje empresarial, no sólo a través del cumplimiento de tareas rutinarias y la homogenización de procesos y productos. Para dinamizar este proceso de capacidades de gestión, administración y control se requiere innovar. Al respecto se preguntó, si en el último año habían realizado en la empresa innovaciones en: el proceso de métodos de manufactura; de entrega o distribución de insumos, y, 
en los sistemas de mantenimiento u operaciones de compra, contabilidad e informáticos. En la figura 4, se puede evidenciar que la mayoría de empresas si realizó innovaciones en los métodos de producción $(82 \%)$ y en los sistemas de mantenimiento para operaciones de compra, contabilidad, computación e informática. Sin embargo, en los procesos de organización de la producción como la distribución de insumos, la mayoría de empresas no realizó innovaciones (52 $\%)$.

\section{Figura 4}

Porcentaje de empresas según tipo de innovaciones organizacionales realizadas

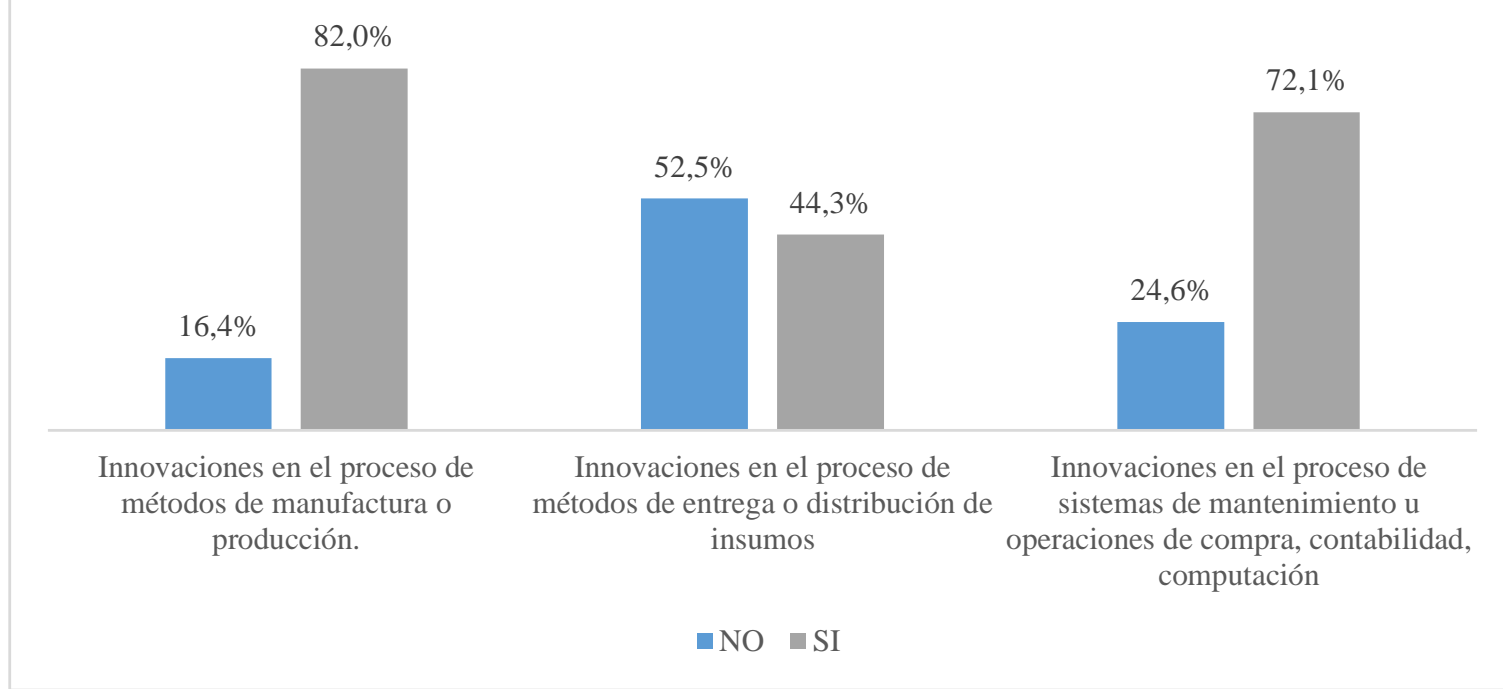

Nota: Elaboración propia a partir de la Encuesta a Empresas Manufactureras de Cuenca, 2020.

A continuación, se presentan los resultados de las variables que han permitido medir como la integración del conocimiento permite obtener resultados de las capacidades organizacionales.

La tabla 6, en la primera columna están las variables analizadas, seguido de la segunda columna que corresponde al promedio simple de las empresas de manufacturas analizadas según el grado de importancia que estas le dieron a cada factor investigado para explicar específicamente las capacidades organizativas de la empresa. La escala aplicada fue de 1 (uno) que significa ninguna importancia hasta 5 (cinco) muy importante. En la tercera columna, se presentan los resultados de la empresa "X", seleccionada porque presenta los mejores resultados obtenidos en todas las variables de análisis. Finalmente, en la cuarta columna se presenta la empresa "Y", cuyos resultados son los peores de todo el sector analizado. Cabe recalcar que no se ha realizado una categorización de las empresas con mejores y peores capacidades organizativas, sino se ha seleccionado el límite superior de la mejor empresa $(\mathrm{X})$ y de la peor $(\mathrm{Y})$.

Entonces, en la tabla 6 se tiene el promedio simple del sector de la manufactura en cuanto a cada factor analizado de capacidades organizacionales y también la mejor empresa $(\mathrm{X})$ y la peor empresa (Y). Por ejemplo, entrar a nuevos mercados o aumentar la participación en un mercado requiere de capacidades administrativas relacionadas con una buena planificación y mercadeo, así 
en promedio las empresas de manufacturas de Cuenca le dan una importancia de 4,4, es decir está entre importante y muy importante para el desarrollo del sector. Como es de esperarse la mejor empresa considera muy importante y la peor empresa también ha considerado este factor muy importante. Por lo que se puede evidenciar que para las empresas de Cuenca la entrada a nuevos mercados o el aumento de la participación en el mercado actual es prioritario.

\section{Tabla 6}

Promedio del sector; y, puntajes de las empresas seleccionadas de la manufactura

\begin{tabular}{|c|c|c|c|}
\hline EMPRESAS & $\begin{array}{c}\text { PROMEDIO } \\
\text { DEL TOTAL DE } \\
\text { MANUFACTURA }\end{array}$ & $\begin{array}{l}\text { Promedio } \\
\text { de la } \\
\text { Empresa } \\
\text { "X" }\end{array}$ & $\begin{array}{l}\text { Promedio } \\
\text { de la } \\
\text { Empresa } \\
\text { "Y" }\end{array}$ \\
\hline 1. Aumento de la gama de bienes o servicios & 4.5 & 5 & 3 \\
\hline 2. Mejora de calidad de bienes o servicios & 4.3 & 5 & 4 \\
\hline $\begin{array}{l}\text { 3. Entrada a nuevos mercados o aumento de la } \\
\text { participación de mercado }\end{array}$ & 4.4 & 5 & 5 \\
\hline $\begin{array}{l}\text { 4. Mejoras en la flexibilidad para producir bienes o } \\
\text { servicios (mejoras en los tiempos) }\end{array}$ & 4.4 & 5 & 5 \\
\hline $\begin{array}{l}\text { 5. Reducción de costos laborales por unidad de } \\
\text { producción }\end{array}$ & 4.2 & 5 & 2 \\
\hline 6. Reducción del impacto ambiental & 4.3 & 5 & 2 \\
\hline 7. Mejoras en la seguridad y salud del trabajador & 4.5 & 5 & 3 \\
\hline $\begin{array}{l}\text { 8. Reducción de costos de materias primas y energía } \\
\text { por unidad de producción }\end{array}$ & 4.3 & 5 & 2 \\
\hline $\begin{array}{l}\text { 9. Mejoras en la capacidad instalada para producir } \\
\text { bienes o servicios (volumen máximo de producción } \\
\text { a alcanzar en un tiempo determinado considerando } \\
\text { la disponibilidad de recursos) }\end{array}$ & 4.2 & 5 & 2 \\
\hline 10. Reemplazo de productos o procesos obsoletos & 4.2 & 5 & 1 \\
\hline
\end{tabular}

Elaboración propia a partir de la encuesta a empresas manufactureras de Cuenca, 2020.

Evidentemente el mejor escenario corresponde a la empresa " $\mathrm{X}$ " que obtuvo el mayor puntaje con 5, que corresponde a darle mayor importancia a cada uno de los aspectos que permiten integrar el conocimiento en la organización y pertenece al sector C10 de Elaboración de Productos Alimenticios, y es una empresa pequeña. 
Esta empresa, se destaca esencialmente del resto del sector, en las siguientes categorías: Reemplazo de productos o procesos obsoletos; y, mejoras en la capacidad instalada para producir bienes o servicios (volumen máximo de producción a alcanzar en un tiempo determinado considerando la disponibilidad de recursos). Por lo que, la empresa "X", ha tendido a innovar procesos de generación de nuevos productos y procesos, utilizando nuevas tecnologías en lo que concierne a adquisición de nueva maquinaria; a la vez la dirección organizacional ha tomado decisiones importantes en cuanto al uso y manejo del capital con el que cuenta, para lo cual la correcta capacitación, manejo y dirección del capital humano ha sido un factor trascendental; ya que por medio de esta estrategia logra utilizar eficientemente los recursos y óptimamente las instalaciones adaptadas en el negocio.

Sin embargo, existen elementos que constituyen fortalezas para el sector y se asemejan a los promedios de la empresa "X"; estas categorías positivas y competitivas del sector en conjunto son: aumento de la gama de bienes o servicios; $y$, mejoras en la flexibilidad para producir bienes o servicios (mejoras en los tiempos). En tanto que la mayor desventaja o debilidad del sector en conjunto, comparado con la empresa " $X$ ", es el reemplazo de los productos o procesos obsoletos. En la figura 5, se puede observar la ubicación de todas las empresas analizadas y particularmente la posición de la mejor (X) y la peor (Y).

\section{Figura 5}

Ranking de las empresas manufactureras de Cuenca según promedio de capacidades organizacionales en una escala de 1 (ninguna) al 5 (muy importantes)

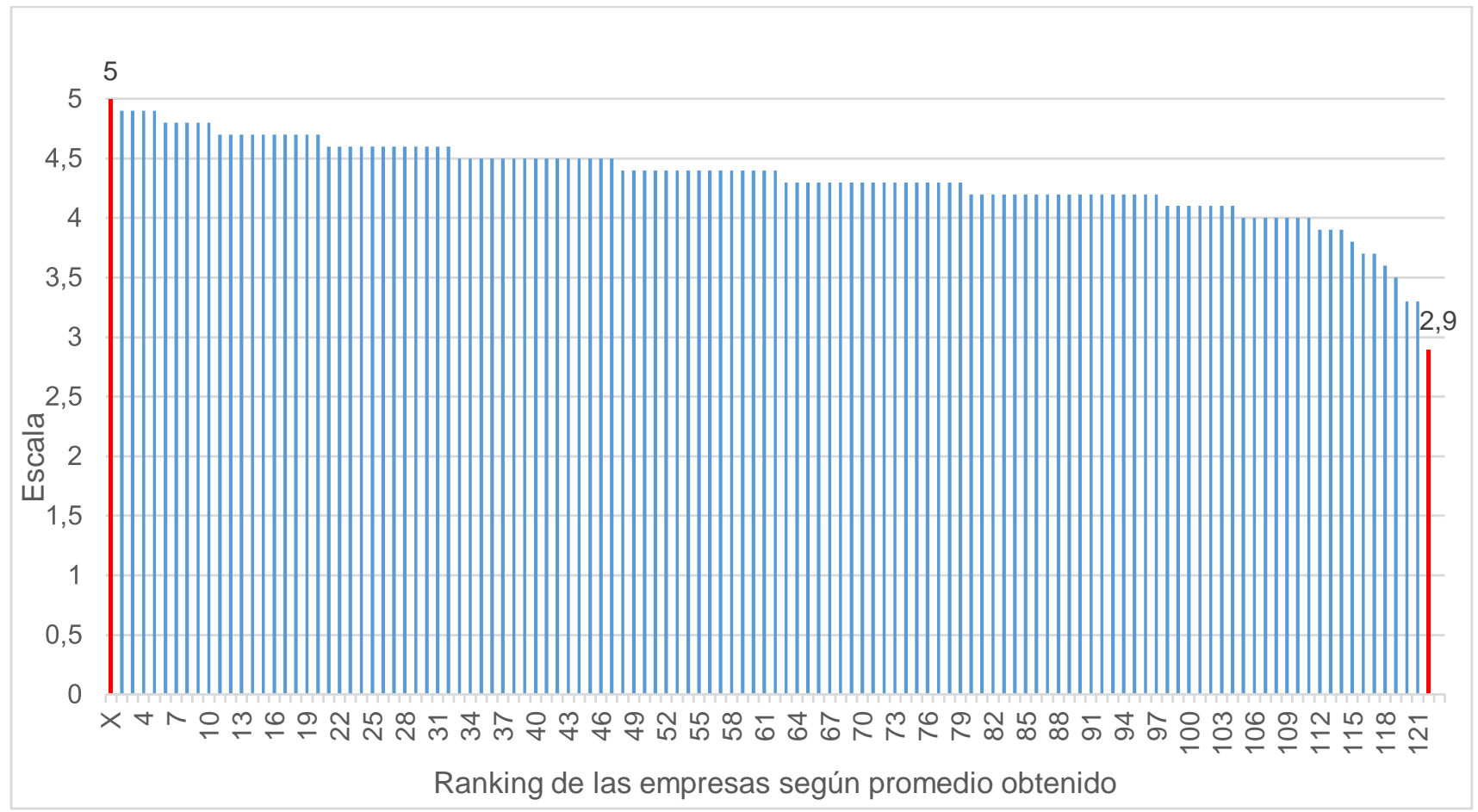

Nota: Elaboración propia a partir de la Encuesta a Empresas Manufactureras de Cuenca, 2020. 
EI peor escenario de la empresa "Y", con 2.9 de puntaje corresponde al promedio simple más bajo del sector de manufacturas y pertenece al subsector C10 de Elaboración de Productos Alimenticios, que corresponde a una microempresa. La figura 6, muestra un panorama de bajo desarrollo de capacidades organizacionales y tecnológicas de la empresa "Y", en relación con los valores medios del total del sector manufacturero.

\section{Figura 6}

Nivel de Desarrollo Organizacional de la Empresa Y, en comparación al promedio de la Manufactura Total (Peor Escenario).

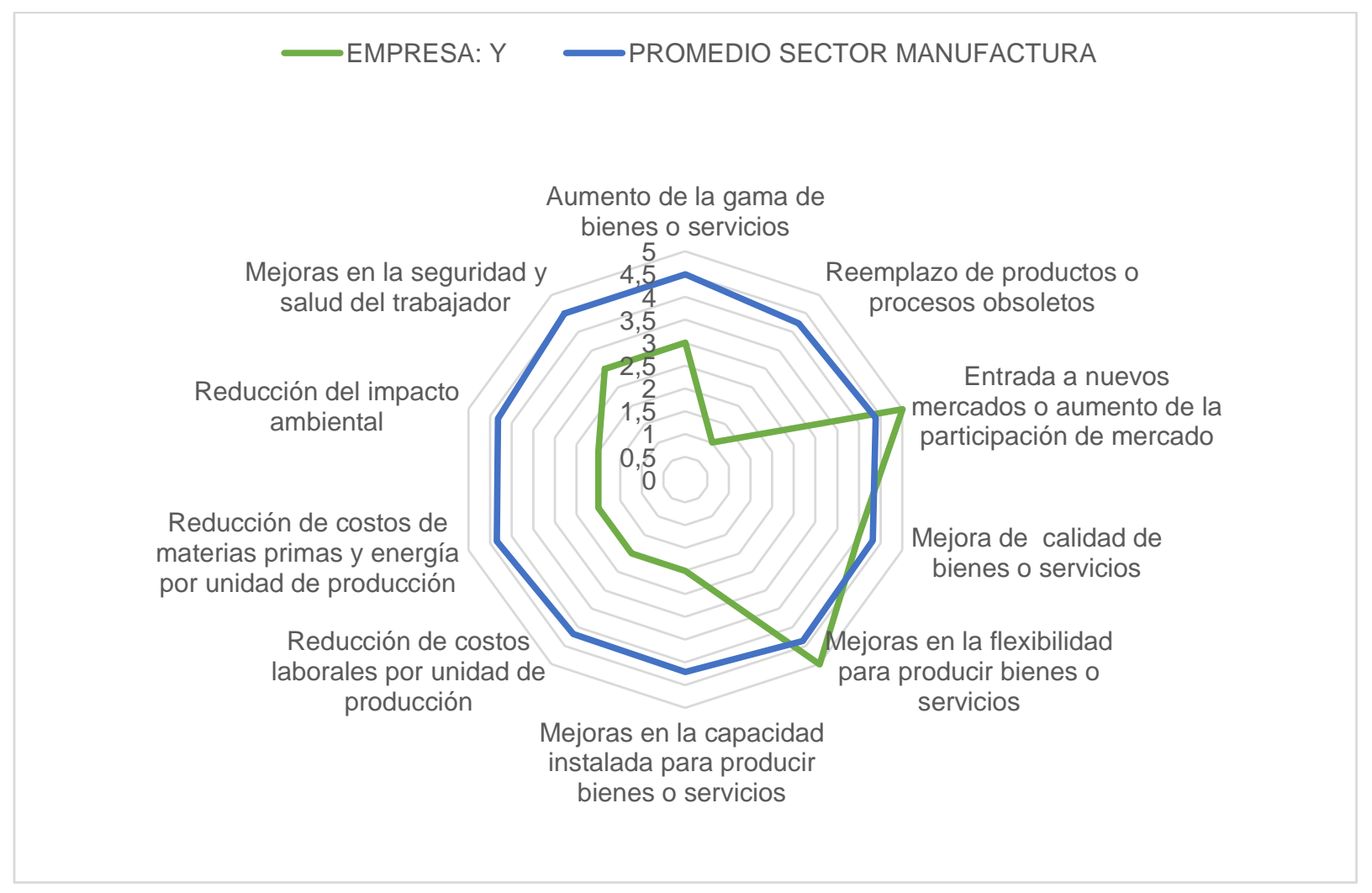

Nota: Elaboración propia a partir de la Encuesta a Empresas Manufactureras de Cuenca, 2020.

Las debilidades de la empresa Y, en comparación con el promedio del sector son: la reducción de costos de materias primas y energía por unidad de producción; la reducción de costos laborales por unidad de producción y las mejoras en las capacidades instaladas. En cambio, las fortalezas son la entrada a nuevos mercados o aumento de la participación de mercado; y, las mejoras en la flexibilidad para producir bienes o servicios (mejoras en los tiempos). Se puede decir entonces que esta empresa tiene un desempeño irregular en los ámbitos analizados, pues les da gran importancia a las estrategias de mercadeo y muy poca a las estrategias de disminución de costos e incrementos de la productividad. 
Con la finalidad de establecer categorías de análisis con las variables estudiadas de las capacidades empresariales se aplicó la metodología estadística de componentes principales. En la tabla 7, se encuentran los resultados obtenidos de la ponderación de cada variable y componente. Es evidente que, las empresas de Cuenca le han dado mayor importancia a las actividades organizativas que les permitan una mayor participación en el mercado o entrar a nuevos mercados o escalar aumentando o diversificando la gama de bienes que ofrecen y realizando mejoras en la capacidad instalada, así este primer componente representa el 43,1\%. La categoría dos relacionada con los cambios tecnológicos de productos y proceso, y la tres con la optimización laboral, están por el rango del $16 \%$ cada una.

\section{Tabla 7}

Resultados del análisis de componentes principales según su ponderación

\begin{tabular}{|c|c|c|c|}
\hline Componente & Variable & $\begin{array}{l}\text { Ponderación } \\
\text { Variable }\end{array}$ & $\begin{array}{l}\text { Ponderación } \\
\text { Componente }\end{array}$ \\
\hline \multirow{3}{*}{$\begin{array}{l}\text { Componente 1: } \\
\text { Capacidad de } \\
\text { ampliación y } \\
\text { mercadeo }\end{array}$} & Aumento de la gama de bienes o servicios & 0.227 & \multirow{3}{*}{0.431} \\
\hline & $\begin{array}{l}\text { Entrada a nuevos mercados o aumento de la } \\
\text { participación de mercado }\end{array}$ & 0.130 & \\
\hline & $\begin{array}{l}\text { Mejoras en la capacidad instalada para producir } \\
\text { bienes o servicios (volumen máximo de producción a } \\
\text { alcanzar en un tiempo determinado considerando la } \\
\text { disponibilidad de recursos) }\end{array}$ & 0.074 & \\
\hline $\begin{array}{c}\text { Componente 2: } \\
\text { Capacidad de } \\
\text { cambios tecnológicos } \\
\text { de productos o } \\
\text { procesos } \\
\end{array}$ & Reemplazo de productos o procesos obsoletos & 0.166 & 0.166 \\
\hline \multirow{2}{*}{$\begin{array}{l}\text { Componente 3: } \\
\text { Capacidad de } \\
\text { optimización laboral }\end{array}$} & $\begin{array}{l}\text { Mejoras en la flexibilidad para producir bienes o } \\
\text { servicios (mejoras en los tiempos) }\end{array}$ & 0.101 & \multirow{2}{*}{0.162} \\
\hline & $\begin{array}{l}\text { Reducción de costos laborales por unidad de } \\
\text { producción }\end{array}$ & 0.062 & \\
\hline $\begin{array}{l}\text { Componentes } 4: \\
\text { Capacidades } \\
\text { relacionadas con }\end{array}$ & $\begin{array}{l}\text { Reducción de costos de materias primas y energía } \\
\text { por unidad de producción }\end{array}$ & 0.056 & \multirow{3}{*}{0.131} \\
\hline $\begin{array}{l}\text { reducción de costos y } \\
\text { mejoras de la }\end{array}$ & Reducción del impacto ambiental & 0.039 & \\
\hline ducción & Mejoras en la seguridad y salud del trabajador & 0.035 & \\
\hline \multirow[t]{2}{*}{$\begin{array}{l}\text { Componente } 5: \\
\text { Capacidad de } \\
\text { mejorar la calidad de } \\
\text { los productos } \\
\end{array}$} & Mejora de calidad de bienes o servicios & 0.110 & 0.110 \\
\hline & Total & 1.000 & 1.000 \\
\hline
\end{tabular}

Elaboración propia a partir de la encuesta a empresas manufactureras de Cuenca, 2020. 
El análisis de componentes principales permitió establecer un índice de las capacidades organizacionales, donde 1.00 o expresado en porcentaje $100 \%$ significa que la empresa le ha dado gran importancia a su operatividad, y en cambio $0.00 \%$ es lo opuesto.

Calculando un promedio ponderado del índice y por tamaño de empresa se obtuvo que en general las capacidades organizacionales o administrativas de las empresas manufactureras de Cuenca son altas. Efectivamente como se observa en la figura 7, el promedio de las empresas cuenta con un índice de capacidades organizacionales de $86.5 \%$. Como era de esperarse, las empresas grandes tienen mejores capacidades $(91.05 \%)$ que las medianas $(85.64 \%)$, pequeñas $(85.07 \%)$ y las micro $(87.47 \%)$.

\section{Figura 7}

Índice de Capacidades Organizacionales de las empresas de Cuenca por tamaño, en porcentaje

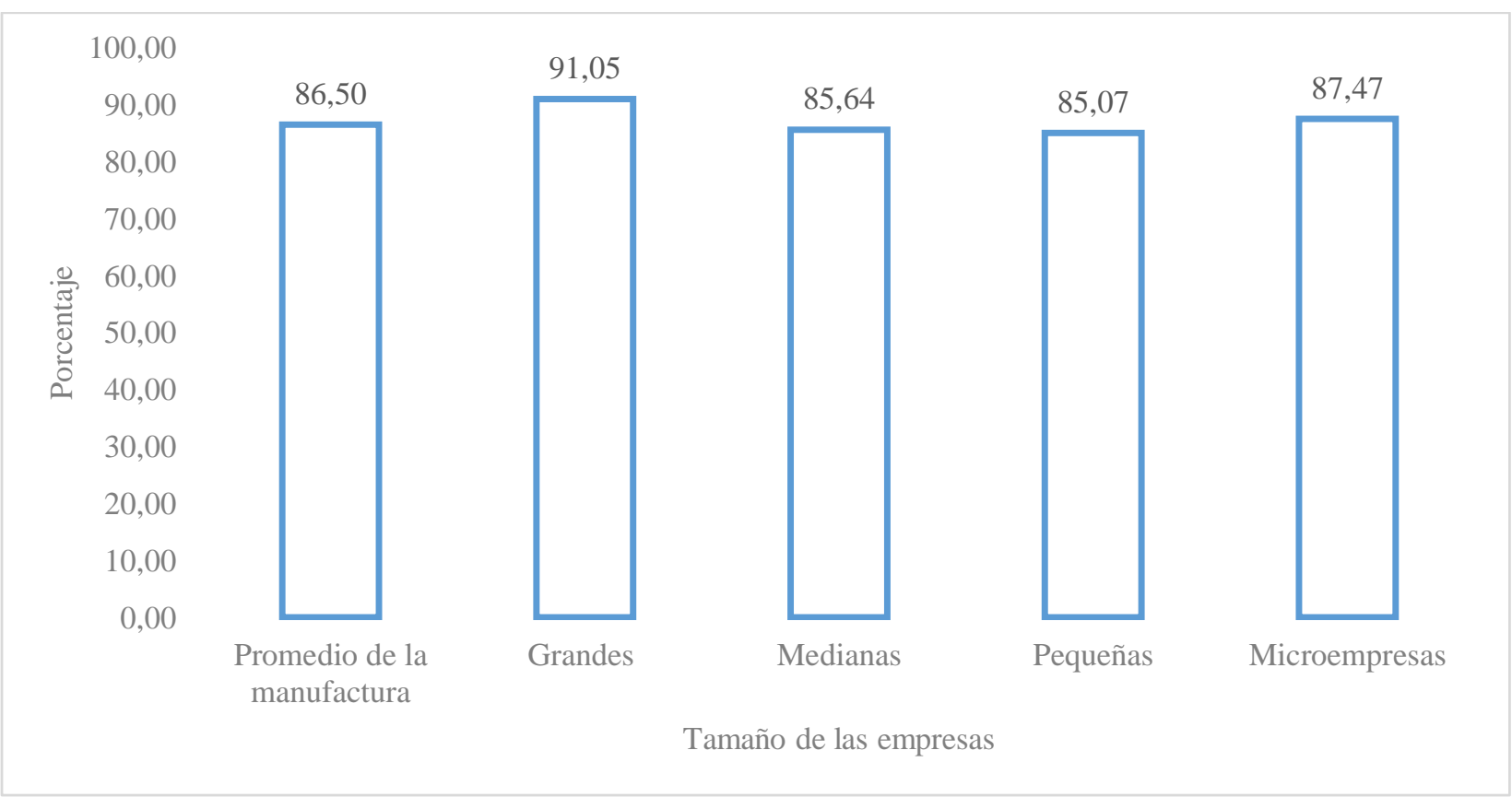

Nota: Elaboración propia a partir de la encuesta a empresas manufactureras de Cuenca, 2020.

\section{Conclusiones}

El objetivo de este trabajo fue conocer las características de las capacidades organizacionales de las empresas de manufacturas de Cuenca en 2019. Así, el análisis en un primer momento, se centró en caracterizar a las capacidades considerando los recursos y el conocimiento (Grant, 1996). Se indagó en las actividades que permiten integrar el conocimiento para lograr resultados en la empresa (Hernández, 2017). Por ejemplo, se consideró a los recursos humanos en lo referido a su formación escolar, experiencia, frecuencia de capacitación anual e inversión en mejorar sus conocimientos y habilidades. 
De esta manera, el estudio evidencia que la mayoría de empresas cuencanas en el 2019 cuentan con directivos con instrucción superior (40.2\%). En cambio, en lo relacionado al personal administrativo y obreros, el nivel de instrucción predominante es la secundaria con $49.2 \%$ y 54.1 $\%$, respectivamente. Aunque las capacidades organizacionales no dependen solo de los recursos será un buen comienzo para que sean sólidas en el largo plazo (Boonpattarakan, 2012). Pues se las concibe también como dinámicas y relacionadas con la cultura organizacional y no se pueden transferir de una empresa a otra sino que dependen de su propio proceso de aprendizaje (Killen et al., 2008).

También se consideró las actividades específicas que realizan las empresas para lograr innovaciones organizacionales y se encontró que $82 \%$ han realizado innovaciones en el proceso de métodos de manufactura o producción y el $72.1 \%$ han aplicado innovaciones en el proceso de sistemas de mantenimiento u operaciones de compra, contabilidad, computación y sistemas informáticos, pero en cambio, solo el $44.3 \%$ ha realizado innovaciones en los procesos de entrega de los productos. Desde luego, la capacidad de innovar y mejorar el proceso productivo les otorga dinamismo a las empresas y de esa manera adaptarse y responder a un entorno cambiante (Acevedo, 2018).

Además, se midió la escala que las empresas han dado a los aspectos de la administración e integración del conocimiento para obtener resultados que permitan mejorar el proceso productivo y la productividad, disminuir los costos, aumentar la gama de bienes y servicios, entrar a nuevos mercados o aumentar la participación en el mercado actual. Se encontró que en la gran variedad de productos que ofrecen las empresas de manufacturas se pueden encontrar empresas que han dado suma importancia a las estrategias organizacionales como es el caso de la empresa " $\mathrm{X}$ ", aunque también se presentan empresas con debilidades sobre todo en el valor que les dan a los sistemas productivos, la productividad y la disminución de costos, como es el caso de la empresa "Y", conforme se puede observar en la tabla 6 de los resultados.

Se puede evidenciar que, las empresas de manufacturas de Cuenca presentan un alto valor de capacidades organizacionales $(86.5 \%$ en promedio) y que las de mayor tamaño muestran mayores fortalezas que las pequeñas y medianas, como se aprecia en la figura 6 .

También se puede decir que el aspecto de gestión, administrativo y organizacional que mayor atención ha recibido es el componente 1 relacionado con la ampliación del mercado o entrar en nuevos mercados.

Finalmente, se tiene que ningún subsector de la manufactura en Cuenca ha consolidado capacidades organizativas. Así, por ejemplo, en el sector de alimentos está la empresa " $X$ " que jerarquiza las estrategias administrativas de los procesos y los resultados que implican la producción, los costos y el mercadeo, y, también la empresa "Y" con debilidades en estos aspectos. Por tal motivo, se considera trascendente ampliar la investigación en lo relacionado a las capacidades tecnológicas y de innovación de las empresas, también en analizar la demanda laboral profundizando en las habilidades y capacidades de los trabajadores y el salario real para mejorar 
el análisis de los costos laborales y, por supuesto, las políticas laborales para mejorar la producción, ya que se trata de una industria donde predomina la fuerza laboral.

\section{Referencias Bibliográficas}

Acevedo, L., \& Albornoz, N. (2019). Theoretical Review of Dynamic Capabilities. Revista Científica Pensamiento y Gestión, 46, 262-283. https://doi.org/10.14482/pege.46.5402

Ambrosini, V., \& Bowman, C. (2009). What are dynamic capabilities and are they a useful construct in strategic management? International Journal of Management Reviews, 11(1), 29-49. https://doi.org/10.1111/j.1468-2370.2008.00251.x

Autio, E., Sapienza, H., \& Almeida, J. (2000). Effects of age at entry, knowledge intensity, and imitability on international growth. The Academy of Management Journal, 43(5), 909-924. http://web.b.ebscohost.com.ezproxy.uct.ac.za/ehost/pdfviewer/pdfviewer?sid=3a60aea1 7e9f-4f90-b175-75087f95aa21\%40sessionmgr114\&vid=1\&hid=107

Boonpattarakan, A. (2012). Model of Thai Small and Medium Sized Enterprises' Organizational Capabilities: Review and Verification. Journal of Management Research, 4(3), 15-42. https://doi.org/10.5296/jmr.v4i3.1557

Carnahan, S., Agarwal, R., Campbell, B. A., \& Franco, A. (2010). The Effect of Firm Compensation Structures on Employee Mobility and Employee Entrepreneurship of Extreme Performers. SSRN Electronic Journal, 1-40. https://doi.org/10.2139/ssrn.1555659

Degravel, D. (2011). Managing organizational capabilities: the Keystone step. Journal of Strategy and Management, 4(3), 251-274. https://doi.org/10.1108/17554251111152270

Ellonen, H. K., Jantunen, A., \& Kuivalainen, O. (2011). The role of dynamic capabilities in developing innovation-related capabilities. International Journal of Innovation Management, 15(3), 459-478. https://doi.org/10.1142/S1363919611003246

García, A., Pineda, D., \& Andrade, M. (2014). Las capacidades tecnológicas para la innovación en empresas de manufactura. Universidad \& Empresa, 17(29), 257-278. https://doi.org/10.12804/rev.univ.empresa.29.2015.11

Grant, R. M. (1996). Prospering in dynamically- Competitive environments: Orginizational capability as knowledge integration. Organization Science, 4(7), 375-387. https://doi.org/10.1016/b978-0-7506-7088-3.50011-5

Helfat, C. E., \& Martin, J. A. (2015). Dynamic Managerial Capabilities: Review and Assessment of Managerial Impact on Strategic Change. Journal of Management, 41(5), 1281-1312. https://doi.org/10.1177/0149206314561301

Helfat, C. E., \& Peteraf, M. A. (2003). The dynamic resource-based view: Capability lifecycles. Strategic Management Journal, 24, 997-1010. https://doi.org/10.1002/smj.332

Hernández, J. (2017). Capacidades tecnológicas y organizacionales de las empresas mexicanas participantes en la cadena de valor de la industria aeronáutica. Economía Teoría y Práctica, 47, 65-98. https://doi.org/10.24275/etypuam/ne/472017/hernandez

Jurksiene, L. y Pundziene, A. (2016), "La relación entre las capacidades dinámicas y la ventaja competitiva de la empresa: el papel mediador de la ambidestreza organizacional". European Business $\quad$ Review, 28(4), 431448. https://doi.org/10.1108/EBR-09-2015-0088

Killen, C. P., Hunt, R. A., \& Kleinschmidt, E. J. (2008). Learning investments and organizational 
capabilities: Case studies on the development of project portfolio management capabilities. International Journal of Managing Projects in Business, 1(3), 334-351. https://doi.org/10.1108/17538370810883800

Knight, G. A., \& Cavusgil, S. T. (2004). Innovation, organizational capabilities, and the bornglobal firm. Journal of International Business Studies, 35(2), 124-141. https://doi.org/10.1057/palgrave.jibs.8400071

Lind, D., Marchal, W., \& Wathen, S. (2012). Estadística aplicada a los negocios y la economía. McGrawHill.

Martínez, G., \& Espinosa, A. (2017). Building of Entrepreneurial and Technological Capacities. The Case of a Small Technology Push Mexican Enterprise. Economía y Desarrollo, 158, $11-128$.

Nelson, R., \& Winter, S. G. (1982). The Schumpeterian Tradeoff Revisited. American Economic Review, 72(1), 114-132.

Nonaka, I., \& Takeuchi, H. (2000). Teoría de la creación del conocimiento organizacional. La organización creadora de conocimiento (pp. 60-103).

Schreyogg, G., \& Kliesch, M. (2007). How dynamic can organizational capabilities be? Towards a dual-process model of capability dynamization. Strategic Management Journal, 28, 913933. https://doi.org/10.1002/smj.613

Superintendencia de Compañías, Valores y Seguros. (2019). Portal de Información. https://www.supercias.gob.ec/portalscvs/

Teece, D., Pisano, G., \& Shuen, A. (1997). Dynamic Capabilities and Strategic Management. Strategic Management Journal, 18(7), 509-533. https://doi.org/10.1176/appi.psychotherapy.2009.63.1.13

Zapata, R. G. J., \& Mirabal, M. A. (2018). Capacidades Dinámicas de la Organización: Revisión de la Literatura y un Modelo Propuesto. Investigación Administrativa, 47(121). http://www.scielo.org.mx/scielo.php?script=sci_abstract\&pid=S2448-

76782018000100005\&lng=es\&nrm=iso\&tlng=es\%0Ahttp://www.redalyc.org/jatsRepo/4 560/456054552003/456054552003.pdf

Zollo, M., \& Winter, S. G. (2002). Deliberate Learning and the Evolution of Dynamic Capabilities. Organization Science, 13(3), 339-351. 